Check for updates

Cite this: J. Mater. Chem. C, 2021 9, 737

DOI: 10.1039/d0tc90273j

rsc.li/materials-c

\section{Correction: Broadband photoelectric tunable quantum dot based resistive random access memory}

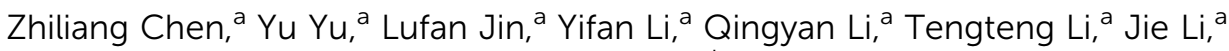 \\ Hongliang Zhao, ${ }^{a}$ Yating Zhang, ${ }^{\text {a }}$ Haitao Dai ${ }^{b}$ and Jianquan Yao ${ }^{a}$
}

Correction for 'Broadband photoelectric tunable quantum dot based resistive random access memory' by Zhiliang Chen et al., J. Mater. Chem. C, 2020, 8, 2178-2185, DOI: 10.1039/C9TC06230K.

The authors regret that the following two corrections are required to this article:

Fig. 1(e) should be removed because it does not represent the actual sample of the lead sulfide quantum dots that was used in this study. Fig. 1(e) is a TEM image of the lead sulfide quantum dots from a previous synthesis. Although the synthesis method was the same, the authors believe this figure (Fig. 1(e)) does not represent the TEM image of lead sulfide quantum dots synthesized this time.

On page 2179 (section 3) of the article, the following sentence should be removed: 'The TEM image in Fig. 1(e) shows that the size distribution of the PbS QDs was uniform and the average diameter of the PbS QDs was approximately $5 \mathrm{~nm}$.'

The results and conclusions are not affected by these errors. The authors regret these errors and apologize for this mistake. The Royal Society of Chemistry apologises for these errors and any consequent inconvenience to authors and readers.

\footnotetext{
${ }^{a}$ Key Laboratory of Opto-Electronics Information Technology (Tianjin University), Ministry of Education, School of Precision Instruments and Opto-Electronics Engineering, Tianjin University, Tianjin 300072, China.E-mail: yating@tju.edu.cn

${ }^{b}$ Tianjin Key Laboratory of Low Dimensional Materials Physics and Preparing Technology, School of Science, Tianjin University, Tianjin, 300072, China
} 